\title{
Growth of InP nanowires on silicon using a thin buffer layer
}

\begin{abstract}
InP nanowires (NWs) are grown on $\mathrm{Si}$ substrate using a thin inter-mediate buffer layer. The buffer layer is grown in two steps. An initial nucleation layer is crucial to accommodate the lattice mismatch between InP and $\mathrm{Si}$. A high quality 2nd layer is grown on this initial layer with smooth morphology suitable for the NW growth. More than $97 \%$ vertical yield is achieved on the buffer layer and the morphology and photoluminescence of the NWs are similar to those grown on $\mathrm{InP}(111) \mathrm{B}$ substrate.
\end{abstract}

Keyword: InP nanowires (NWs); Silicon; Thin inter-mediate buffer layer; III-V materials; Monolithic integration of photonics 